\title{
Decreased expression of long non-coding RNA LOC728290 in human hepatocellular carcinoma
}

\author{
SHAO-GENG ZHANG ${ }^{1}$, YU-FENG LI ${ }^{2}$, NA-NA ZHAO ${ }^{1}$, CHENG-CAI LAI $^{3}$, SI-JIE CHENG ${ }^{1}$, \\ JIN YAN $^{1}$, PEIRUI ZHANG ${ }^{1}$, ZHAOHAI WANG ${ }^{1}$, XI-LIANG WANG ${ }^{3}$ and PENG-HUI YANG ${ }^{1,3}$ \\ ${ }^{1}$ Department of Hepatobiliary, 302 Military Hospital of China, Beijing 100039; ${ }^{2}$ Institute of Geriatric Cardiology, \\ Chinese PLA General Hospital, Beijing $100853 ;{ }^{3}$ State Key Laboratory of Pathogen and Biosecurity, \\ Beijing Institute of Microbiology and Epidemiology, Beijing 100071, P.R. China
}

Received June 21, 2016; Accepted June 2, 2017

DOI: $10.3892 / 01.2017 .6776$

\begin{abstract}
Hepatocellular carcinoma (HCC) is a leading cause of cancer-associated mortality worldwide. Despite progress in the diagnosis and treatment of HCC, prognosis remains unfavorable. Long non-coding RNAs (lncRNAs) are emerging as important factors in tumorigenesis and cancer progression; however, the underlying molecular mechanisms and clinical significance of lncRNAs in HCC remain largely unknown. The present study examined the expression pattern and clinical significance of a novel lncRNA, LOC728290, in HCC. Expression of LOC728290 was markedly decreased in HCC tissues compared with adjacent non-tumor liver tissues, as detected using the reverse transcription-quantitative polymerase chain reaction (RT-qPCR). The area under the receiver operating characteristic curve for LOC728290 was 0.728 . The expression of LOC728290 was associated with the level of $\alpha$-fetoprotein and microvascular invasion. Furthermore, patients with low LOC728290 expression exhibited decreased recurrence-free survival times $(\mathrm{P}<0.05)$ compared with those with high LOC728290 expression. The results of the present study indicated that downregulation of LOC728290 in patients with HCC may be a powerful tumor biomarker, with potential clinical applications in prognosis as well as a therapeutic target.
\end{abstract}

\section{Introduction}

Hepatocellular carcinoma (HCC) is characterized by high morbidity and mortality, and is one of the most prevalent malignancies worldwide (1). Although progress has been

Correspondence to: Dr Peng-Hui Yang, Department of Hepatobiliary, 302 Military Hospital of China, 100 West Fourth Ring Road, Beijing 100039, P.R. China

E-mail: ypenghuiamms@hotmail.com

Key words: long non-coding RNA, hepatocellular carcinoma, LOC728290, reverse transcription-quantitative polymerase chain reaction made in the diagnosis and treatment of $\mathrm{HCC}$, the prognosis for patients with HCC remains poor due to resistance to conventional chemotherapy and radiotherapy $(2,3)$. Following surgical treatment, $>60 \%$ of patients experience recurrence and metastasis within 1 year (4). Biomarker-based tumor recognition has promise for condition assessments that guide treatment; however, currently, few biomarkers have been applied in clinical practice (5). Therefore, it remains important to identify novel biomarkers for early diagnosis and prognosis evaluation for patients with HCC.

Long non-coding RNAs (lncRNAs) are >200 nucleotides in length and do not code for, but may interact with, proteins (6). Although not as well-characterized as small non-coding RNAs, including microRNAs, IncRNAs serve important roles in the regulation of a variety of cellular processes, including stem cell pluripotency, cell growth, cell proliferation, apoptosis, metabolism and cancer cell migration (7-12). Functional lncRNAs may be useful in cancer diagnosis and prognosis and may serve as potential therapeutic targets. Tang et al (13) reported that three lncRNAs (RP11-160H22.5, XLOC_014172 and LOC149086) were upregulated in HCC relative to cancer-free controls. Furthermore, XLOC_014172 and LOC149086 were confirmed to be markedly increased in metastatic HCC. In addition, levels of these three lncRNAs were identified to be decreased following cancer resection in the majority of patients. Xu et al (14) identified an lncRNA, LALR1, that was involved in liver regeneration. Yuan et al (15) observed that IncRNA-activated by transforming growth factor $\mathrm{b}$ upregulation in $\mathrm{HCC}$, modulated tumorigenesis and progression. Wang et al (16) identified that the oncofetal lncRNA PVT1 promoted proliferation and stem cell-like properties in HCC cells, suggesting that this lncRNA may be involved in HCC progression.

In the present study, microarray data from the human IncRNA datasets GSE55191 and GSE5804 were analyzed, and it was identified that lncRNA LOC728290 expression levels in HCC tissues were significantly decreased compared with adjacent non-tumor tissues $(\mathrm{P}<0.05)$. Distinct LOC728290 expression levels in $\mathrm{HCC}$ and paired adjacent non-tumor samples were then validated using the reverse transcription-quantitative polymerase chain reaction (RT-qPCR). Furthermore, the association between LOC728290 expression 
levels, clinicopathological characteristics and recurrence-free survival (RFS) times in patients with HCC were analyzed to determine whether lncRNA LOC728290 may be a useful diagnostic and prognostic indicator in HCC.

\section{Materials and methods}

Patients and specimens. Data from 65 consecutive patients (51 males and 14 females), who underwent surgery for HCC at 302 Beijing Hospital (Beijing, China), between August 2013 and April 2016, were accessed from the records of the Department of Hepatobiliary Surgery. None of the patients had received preoperative chemotherapy or radiation therapy. All HCC diagnoses were confirmed histopathologically by a clinical pathologist. Tumor tissues and adjacent non-tumor tissue specimens were collected from the patients subsequent to obtaining written informed consent, in accordance with the institutional guidelines of 302 Beijing Hospital's Ethics Committee. Resected tumor tissue and adjacent normal tissue specimens were immediately snap-frozen in liquid nitrogen and stored in a tissue bank until use. The experimental operators were blinded to the clinical data. Patient characteristics are presented in Table I.

$R N A$ preparation, reverse transcription and $R T-q P C R$. RNA from frozen HCC tissues and adjacent non-tumor tissues $(n=65)$ was extracted using TRIzol reagent (Thermo Fisher Scientific, Inc., Waltham, MA, USA), according to the manufacturer's protocol. RNA integrity was evaluated using a NanoDrop ND-1000 spectrophotometer (NanoDrop Technologies; Thermo Fisher Scientific, Inc.) and cDNA was synthesized from 50 ng total RNA for each sample. LOC728290 expression levels were quantified using RT-qPCR performed on an ABI 7500 system (Applied Biosystems; Thermo Fisher Scientific, Inc.) using Maxima SYBR-Green RT-qPCR master mix (Thermo Fisher Scientific, Inc.), following the manufacturers' protocols. GAPDH expression was monitored as the endogenous control, and all samples were normalized to human GAPDH. All reactions were run in triplicate, using LOC728290-specific primers designed and synthesized by Sangon Biotech Co., Ltd. (Shanghai, China). Primer sequences were as follows: LOC728290 5'-AAAGCACAGGTGACTGTA ACAC-3' (forward) and 5'-TGGGCATTCTCATCGCAGTC-3' (reverse), and GAPDH 5'-CAGCCTCAAGATCATCAGCA-3' (forward) and 5'-TGTGGTCATGAGTCCTTCCA-3' (reverse). The amplification profile was $95^{\circ} \mathrm{C}$ for $5 \mathrm{~min}$, followed by 40 cycles of denaturation at $95^{\circ} \mathrm{C}$ for $15 \mathrm{sec}$ and annealing at $60^{\circ} \mathrm{C}$ for $30 \mathrm{sec}$. The median of triplicate reactions was used to calculate relative 1 ncRNA expression $(\triangle \mathrm{Cq}=\mathrm{Cq}$ median lncRNA-Cq median GAPDH). Expression fold changes were calculated using the $2^{-\Delta \Delta \mathrm{Cq}}$ method (17).

Statistical analysis. Statistically significant differences between groups were determined using two-tailed Student's t-tests. A receiver operating characteristic (ROC) curve was plotted to determine the discrimination of the expression level of LOC728290 discriminated between HCC tissues and adjacent non-tumor tissues. Associations between LOC728290 expression and clinicopathological characteristics were analyzed using one-way analysis of variance with
Bonferroni correction. The association between RFS times and LOC728290 expression levels in patients with HCC was analyzed using Kaplan-Meier estimator analysis. All statistical analyses were performed using SPSS for Windows software (version 16.0; SPSS Inc., Chicago, IL, USA). P<0.05 was considered to indicate a statistically significant difference.

\section{Results}

lncRNA LOC728290 is downregulated in HCC tissues relative to adjacent non-tumor tissues. To assess the potential clinical significance of LOC728290, the expression level in HCC tissues and adjacent non-tumor tissues was analyzed using RT-qPCR. LOC728290 expression was significantly downregulated in $83.0 \%$ of tumors $(54 / 65$; fold change $\geq 1.0)$, relative to adjacent non-tumor tissues $(\mathrm{P}<0.05$; Fig. $1 \mathrm{~A}$ and $\mathrm{B})$. A ROC analysis was performed to evaluate the ability of the LOC728290 expression to discriminate between the tumor and control samples. The total area under the curve (AUC) for LOC728290 was 0.728 (Fig. 1C), suggesting that the LOC728290 level has adequate sensitivity and specificity to discriminate between HCC tissues and adjacent non-tumor tissues.

IncRNA LOC728290 expression is associated with $\alpha$-fetoprotein (AFP) levels and with microvascular invasion in patients with HCC. To determine whether LOC728290 expression in HCC tissue was associated with clinicopathological parameters, AFP levels and the presence of microvascular invasion in samples from patients with HCC were examined. The AFP level is a critical tumor biomarker for patients with HCC. LOC728290 expression was decreased in tissue samples where serum AFP was $\geq 20 \mathrm{ng} / \mathrm{ml}$ (Fig. 2A). In addition, decreased LOC728290 expression was exhibited in patients with microvascular invasion compared with those without (Fig. 2B).

Association between lncRNA LOC728290 expression and clinicopathological features of patients with HCC. To further analyze the association between lncRNA expression and clinicopathological parameters, the 65 patients with $\mathrm{HCC}$ were divided into high-(n=32) and low-(n=33) LOC728290 expression groups, according to the mean value of LOC728290 expression in their tumor tissues. As presented in Table I, whereas the low-LOC728290 expression group exhibited increased serum AFP levels $(\mathrm{P}<0.05)$ and microvascular invasion $(\mathrm{P}<0.01)$ compared with the high-LOC728290 group, no significant association was identified between LOC728290 expression and other clinicopathological features including age, sex, tumor size, clinical stage, histological grade, alcohol consumption, smoking status, hepatitis B virus (HBV), recurrence, portal vein tumor thrombosis (PVTT) or liver cirrhosis.

LOC728290 expression is associated with RFS times in HCC. Kaplan-Meier estimator and log-rank analyses were performed to examine the association between levels of LOC728290 expression and patient survival. As presented in Table II, LOC728290 expression, PVTT, tumor size and microvascular invasion were significantly associated with RFS times. In particular, patients with a low level of LOC728290 expression 
Table I. Association of long non-coding RNA LOC728290 expression with clinicopathological characteristics of patients with hepatocellular carcinoma.

\section{LOC728290 expression}

\begin{tabular}{|c|c|c|c|c|}
\hline Characteristic & Total & Low & High & P-value \\
\hline Sex & & & & 0.203 \\
\hline Male & 51 & 28 & 23 & \\
\hline Female & 14 & 5 & 9 & \\
\hline Age, years & & & & 0.924 \\
\hline$<60$ & 41 & 21 & 20 & \\
\hline$\geq 60$ & 24 & 12 & 12 & \\
\hline Tumor size, $\mathrm{cm}$ & & & & 0.267 \\
\hline$<5$ & 28 & 12 & 16 & \\
\hline$\geq 5$ & 37 & 21 & 16 & \\
\hline $\mathrm{AFP}, \mathrm{ng} / \mathrm{ml}$ & & & & $0.033^{\mathrm{a}}$ \\
\hline$<20$ & 26 & 9 & 17 & \\
\hline$\geq 20$ & 39 & 24 & 15 & \\
\hline Histological grade & & & & 0.136 \\
\hline Well & 2 & 2 & 0 & \\
\hline Moderately/poorly & 56 & 26 & 30 & \\
\hline Clinical stage & & & & 0.236 \\
\hline I and II & 47 & 26 & 21 & \\
\hline III and IV & 18 & 7 & 11 & \\
\hline Tumor number & & & & 0.087 \\
\hline Solitary & 54 & 30 & 24 & \\
\hline Multiple & 11 & 3 & 8 & \\
\hline Alcohol consumption & & & & 0.267 \\
\hline Yes & 30 & 13 & 17 & \\
\hline No & 35 & 20 & 15 & \\
\hline Smoking status & & & & 0.165 \\
\hline Yes & 23 & 9 & 14 & \\
\hline No & 42 & 24 & 18 & \\
\hline $\mathrm{HBV}$ & & & & 0.156 \\
\hline Yes & 39 & 17 & 22 & \\
\hline No & 26 & 16 & 10 & \\
\hline Recurrence & & & & 0.337 \\
\hline Yes & 22 & 13 & 9 & \\
\hline No & 43 & 20 & 23 & \\
\hline PVTT & & & & 0.172 \\
\hline Yes & 33 & 14 & 19 & \\
\hline No & 32 & 19 & 13 & \\
\hline Microvascular invasion & & & & $0.022^{\mathrm{a}}$ \\
\hline Yes & 47 & 28 & 19 & \\
\hline No & 18 & 5 & 13 & \\
\hline Liver cirrhosis & & & & 0.221 \\
\hline Absence & 35 & 14 & 21 & \\
\hline Presence & 25 & 14 & 11 & \\
\hline
\end{tabular}

Low expression of LOC728290 was identified to be significantly associated with microvascular invasion ( $\mathrm{P}=0.022)$ and serum $\mathrm{AFP}(\mathrm{P}=0.033)$. However, LOC728290 expression was not significantly associated with sex, age, tumor size, liver cirrhosis, histological grade, alcohol consumption, smoking status, HBV, recurrence, PVTT and clinical stage (P>0.05). AFP, $\alpha$-fetoprotein; HBV, hepatitis B virus; PVTT, portal vein tumor thrombosis. ${ }^{a} \mathrm{P}<0.05$. 
A

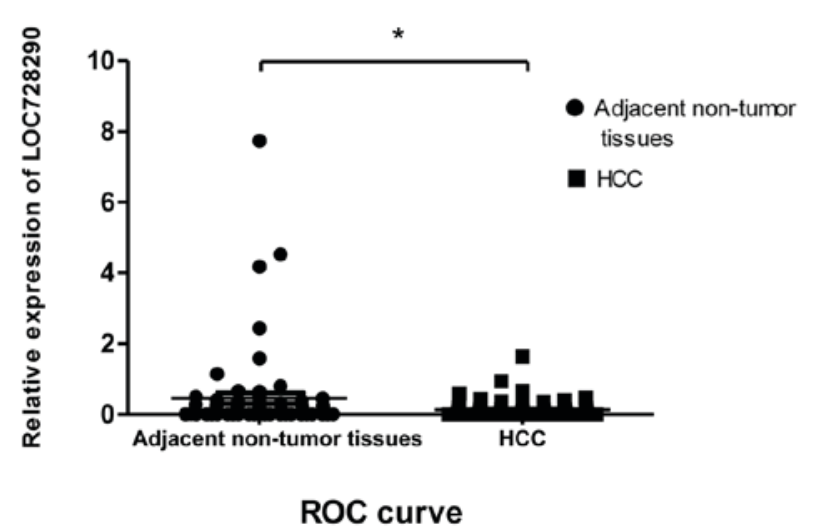

B

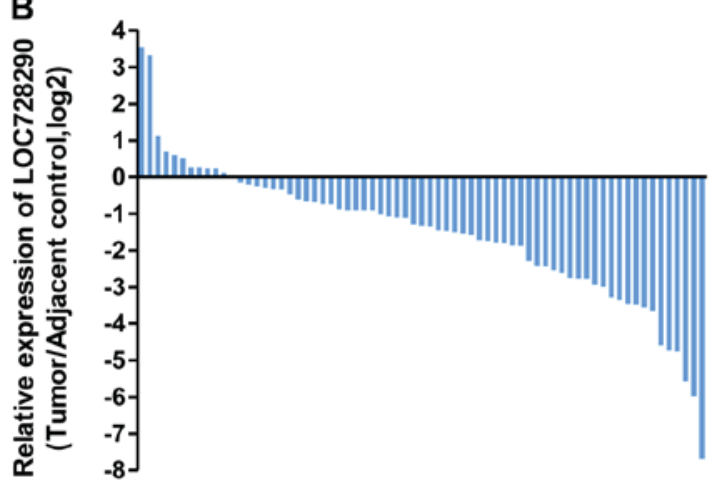

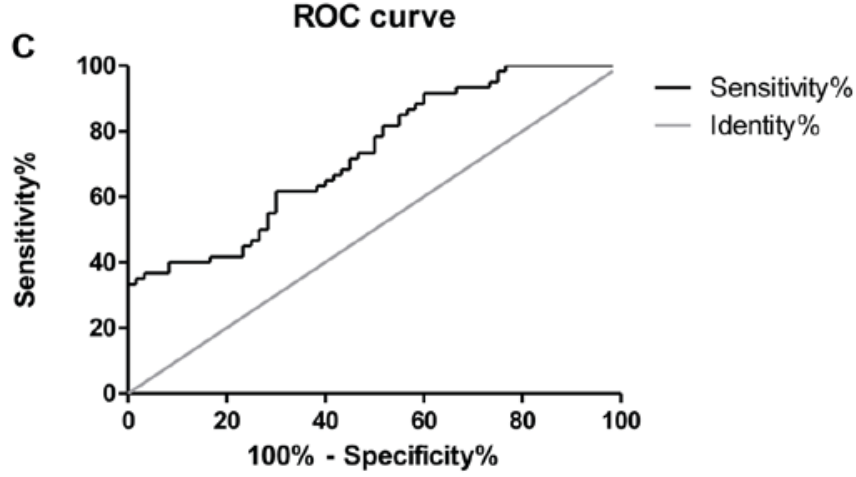

Figure 1. Relative expression of long non-coding RNA LOC728290 in patients with HCC. (A) Lower relative LOC728290 levels were exhibited in HCC tissues compared with adjacent non-tumor tissues from patients. (B) LOC728290 expression was classified into two groups: Positive values indicate higher LOC728290 expression in tumor tissue compared with non-tumor tissue; negative values indicate lower LOC728290 expression in tumor tissue. (C) The area under the receiver operating characteristic curve was 0.728 , distinguishing HCC from adjacent normal tissues. ${ }^{*} \mathrm{P}<0.05$. HCC, hepatocellular carcinoma.

A

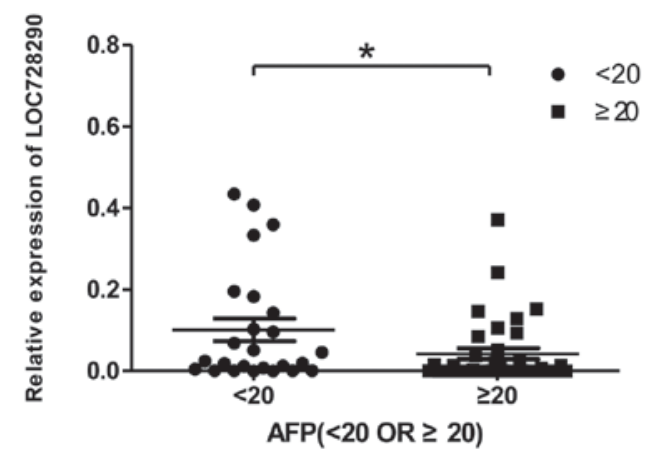

B

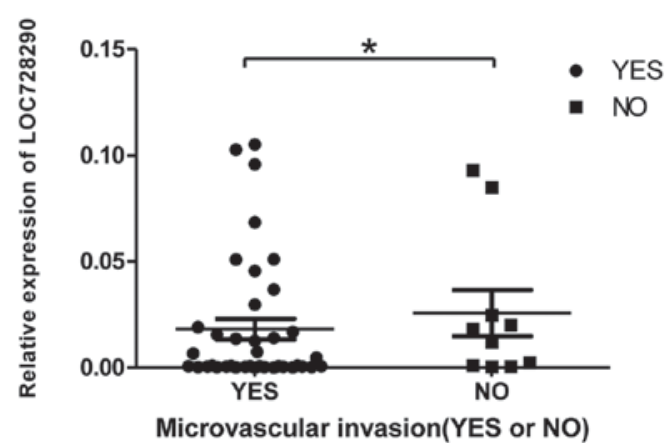

Figure 2. Long non-coding RNA LOC728290 expression is associated with serum AFP levels and microvascular invasion. (A) Comparison of the relative levels of LOC728290 between serum AFP levels $<20 \mathrm{ng} / \mathrm{ml}$ and AFP $\geq 20 \mathrm{ng} / \mathrm{ml}$ in patients with HCC. (B) Comparison between the relative levels of LOC728290 with and without microvascular invasion in patients with HCC. "P<0.05. AFP, $\alpha$-fetoprotein; HCC, hepatocellular carcinoma.

exhibited significantly decreased RFS times ( $\mathrm{P}=0.023$; Fig. 3) compared with patients with high LOC728290 expression. Significantly decreased RFS times were also observed in patients with PVTT $(\mathrm{P}<0.05)$, tumors $\geq 5 \mathrm{~cm}(\mathrm{P}<0.001)$ or microvascular invasion $(\mathrm{P}<0.001)$. Thus, these data indicate that low expression of LOC728290 may indicate a poorer prognosis for patients with HCC.

\section{Discussion}

$\mathrm{HCC}$ is one of the most common malignant tumors in China; its incidence and resulting mortality have increased annually (18).
The mechanisms of occurrence and development of HCC are complex and involve altered activity of a number of oncogenes and tumor suppressor genes; abnormal expression of lncRNAs has been demonstrated to serve an important role in the processes of invasion and metastasis (19-21). Previous studies have revealed that the IncRNAs H19, MALAT1 and HULC are important in HCC progression (22-24); however, only a limited number studies have addressed the biological function and clinical significance of lncRNAs in HCC.

In the present study, differential expression of a novel lncRNA, LOC728290, between HCC tissue and adjacent non-tumor tissues from patients with HCC was identified. 
Table II. Univariate analysis of RFS times for the 65 patients with hepatocellular carcinoma studied.

\begin{tabular}{lcc}
\hline Variable & $\mathrm{n}$ & P-value \\
\hline Sex & & 0.33 \\
$\quad$ Male & 51 & \\
Female & 14 & \\
Age, years & & 0.302 \\
$\quad<60$ & 41 & \\
$\geq 60$ & 24 &
\end{tabular}

Tumor size, $\mathrm{cm}$

$0.0067^{\mathrm{b}}$

$<5$

$\geq 5$

37

AFP, ng/ml

$<20$

$\geq 20$

Histological grade

Well

Moderately/poorly

56

Clinical stage

I and II

III and IV

18

Tumor number

Solitary

Multiple

54

11

Alcohol consumption

Yes

No

Smoking status

Yes

No

HBV

Yes

No

PVTT

Yes

No

Microvascular invasion

Yes

No

18

Liver cirrhosis

Absence
Presence
LOC728290 expression
Low
High

Decreased RFS times were identified to be significantly associated with microvascular invasion, PVTT and low expression of LOC728290. However, RFS times were not significantly associated with sex, age, tumor size, liver cirrhosis, histological grade, drinking state, smoking state, HBV and clinical stage. ${ }^{a} \mathrm{P}<0.05 ;{ }^{b} \mathrm{P}<0.01$. RFS, recurrence-free survival; AFP, $\alpha$-fetoprotein; HBV, hepatitis B virus; PVTT, portal vein tumor thrombosis.

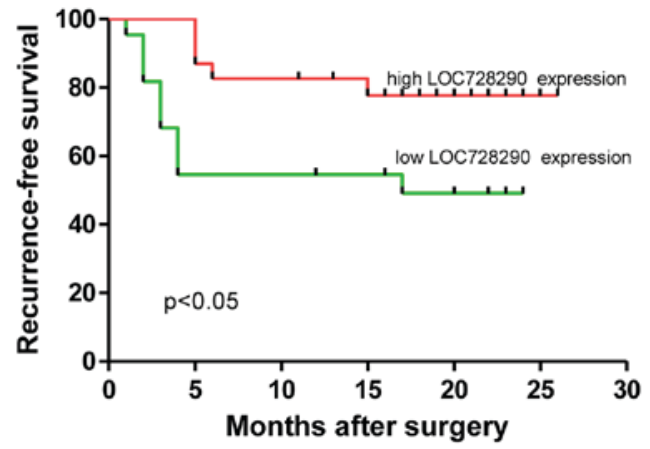

Figure 3. Kaplan-Meier estimator curves for recurrence-free survival in patients with hepatocellular carcinoma with low or high expression of LOC728290.

Results demonstrated that LOC728290 expression in HCC was significantly decreased compared with non-tumor tissue. The ROC AUC of LOC728290 was 0.728, demonstrating specificity and sensitivity in the diagnosis of HCC. Patients with HCC with low levels of LOC728290 expression exhibited significantly decreased RFS times $(\mathrm{P}=0.047)$ compared with patients with high expression. Furthermore, patients with HCC with PVTT $(\mathrm{P}<0.05)$, tumor size $\geq 5 \mathrm{~cm}(\mathrm{P}<0.001)$ and microvascular invasion $(\mathrm{P}<0.001)$ exhibited significantly decreased RFS times $(\mathrm{P}<0.001)$, respectively, which initially revealed the prognostic value of LOC728290. Overall, LOC728290 may serve an important role in the development and progression of HCC.

It has been reported that aberrant lncRNA expression results in dysregulation of downstream effectors and that lncRNAs may serve essential roles in numerous biological functions leading to HCC, as lncRNAs including HOTAIR, H19 and ZEB1-AS1 have been identified to be potential prognostic indicators in a number of tumors (25-27).

In the present study, the association between LOC728290 expression and clinicopathological characteristics of patients with HCC was analyzed and a significant negative association was revealed between LOC728290 expression and serum AFP levels. Determination of serum AFP levels is essential in the clinical diagnosis of liver cancer $(28,29)$. Thus, the results of the present study led to the hypothesis that LOC728290 may be a promising biomarker for HCC. In addition, the results of the present study revealed that decreased LOC728290 expression was associated with microvascular invasion, which, along with PVTT, is a primary mechanism of extrahepatic metastasis in HCC $(30,31)$. Results from the present study indicated that LOC728290 may serve an important role in the development of HCC and that an investigation of possible mechanisms is warranted. However, no significant association was revealed between LOC728290 expression and other clinicopathological features, including age, sex, tumor size, clinical stage, histological grade, alcohol consumption, smoking status, HBV infection, tumor recurrence, PVTT or liver cirrhosis. Much larger clinical cohorts and extended follow-up times are required to confirm the results of the present study. Further in vitro and in vivo experiments are required to investigate the function of lncRNA LOC728290 in HCC and to elucidate its role in the underlying molecular mechanisms of HCC onset and progression. 
To the best of our knowledge, the results of the present study identified for the first time that lncRNA LOC728290 levels were significantly decreased in HCC tissues and that downregulation of IncRNA LOC728290 was positively associated with increased serum AFP levels and microvascular invasion in patients with HCC. Patients with HCC with low levels of LOC728290 expression demonstrated significantly decreased RFS times compared with patients with higher expression. These results demonstrate that lncRNA LOC728290 may exhibit potential as a diagnostic and prognostic biomarker for HCC.

\section{References}

1. Tsochatzis EA, Meyer T and Burroughs AK: Hepatocellular carcinoma. N Engl J Med 366: 92-93, 2012.

2. Llovet JM, Burroughs A and Bruix J: Hepatocellular carcinoma. Lancet 362: 1907-1917, 2003.

3. Maluccio M and Covey A: Recent progress in understanding, diagnosing, and treating hepatocellular carcinoma. CA Cancer J Clin 62: 394-399, 2012.

4. Zimmerman MA, Ghobrial RM, Tong MJ, Hiatt JR, Cameron AM, Hong J and Busuttil RW: Recurrence of hepatocellular carcinoma following liver transplantation: A review of preoperative and postoperative prognostic indicators. Arch Surg 143: 182-188, 2008

5. Ludwig JA and Weinstein JN: Biomarkers in cancer staging, prognosis and treatment selection. Nat Rev Cancer 5: 845-856, 2005.

6. Esteller M: Non-coding RNAs in human disease. Nat Rev Genet 12: 861-874, 2011.

7. Kung JT, Colognori D and Lee JT: Long noncoding RNAs: Past, present, and future. Genetics 193: 651-669, 2013.

8. Wang J, Xie G, Singh M, Ghanbarian AT, Raskó T, Szvetnik A, Cai H, Besser D, Prigione A, Fuchs NV, et al: Primate-specific endogenous retrovirus-driven transcription defines naive-like stem cells. Nature 516: 405-409, 2014

9. Wang Y, Wang Y, Li J, Zhang Y, Yin H and Han B: CRNDE, a long-noncoding RNA, promotes glioma cell growth and invasion through mTOR signaling. Cancer Lett 367: 122-128, 2015.

10. Leveille N, Melo CA, Rooijers K, Díaz-Lagares A, Melo SA Korkmaz G, Lopes R, Akbari Moqadam F, Maia AR, Wijchers PJ, et al: Genome-wide profiling of p53-regulated enhancer RNAs uncovers a subset of enhancers controlled by a IncRNA. Nat Commun 6: 6520, 2015.

11. Qiu JJ, Wang Y, Ding JX, Jin HY, Yang G and Hua KQ: The long non-coding RNA HOTAIR promotes the proliferation of serous ovarian cancer cells through the regulation of cell cycle arrest and apoptosis. Exp Cell Res 333: 238-248, 2015.

12. Cui M, Xiao Z, Wang Y, Zheng M, Song T, Cai X, Sun B, Ye L and Zhang X: Long noncoding RNA HULC modulates abnormal lipid metabolism in hepatoma cells through an miR-9-mediated RXRA signaling pathway. Cancer Res 75: 846-857, 2015.

13. Tang J, Jiang R, Deng L, Zhang X, Wang K and Sun B: Circulation long non-coding RNAs act as biomarkers for predicting tumorigenesis and metastasis in hepatocellular carcinoma. Oncotarget 6: 4505-4515, 2015 .

14. Xu D, Yang F, Yuan JH, Zhang L, Bi HS, Zhou CC, Liu F, Wang $\mathrm{F}$ and Sun SH: Long noncoding RNAs associated with liver regeneration 1 accelerates hepatocyte proliferation during liver regeneration by activating $\mathrm{Wnt} / \beta$-catenin signaling. Hepatology 58: 739-751, 2013.

15. Yuan JH, Yang F, Wang F, Ma JZ, Guo YJ, Tao QF, Liu F, Pan W, Wang TT, Zhou CC, et al: A long noncoding RNA activated by TGF- $\beta$ promotes the invasion-metastasis cascade in hepatocellular carcinoma. Cancer Cell 25: 666-681, 2014.
16. Wang F, Yuan JH, Wang SB, Yang F, Yuan SX, Ye C, Yang N, Zhou WP, Li WL, Li W and Sun SH: Oncofetal long noncoding RNA PVT1 promotes proliferation and stem cell-like property of hepatocellular carcinoma cells by stabilizing NOP2 Hepatology 60: 1278-1290, 2014

17. Livak KJ and Schmittgen TD: Analysis of relative gene expression data using real-time quantitative PCR and the 2(-Delta Delta C(T)) method. Methods 25: 402-408, 2001.

18. Lechel A and Gougelet A: Early HCC treatment: A future strategy against interferon/miR-484 axis to revert precancerous lesions? Gut 65: 1073-1074, 2016.

19. Iguchi T, Uchi R, Nambara S, Saito T, Komatsu H, Hirata H, Ueda M, Sakimura S, Takano Y, Kurashige J, et al: A long noncoding RNA, lncRNA-ATB, is involved in the progression and prognosis of colorectal cancer. Anticancer Res 35: 1385-1388, 2015.

20. Qiu JJ, Lin YY, Ding JX, Feng WW, Jin HY and Hua KQ: Long non-coding RNA ANRIL predicts poor prognosis and promotes invasion/metastasis in serous ovarian cancer. Int J Oncol 46: 2497-2505, 2015

21. Yue B, Qiu S, Zhao S, Liu C, Zhang D, Yu F, Peng Z and Yan D: LncRNA-ATB mediated E-cadherin repression promotes the progression of colon cancer and predicts poor prognosis. J Gastroenterol Hepatol 31: 595-603, 2016.

22. Luo F, Sun B, Li H, Xu Y, Liu Y, Liu X, Lu L, Li J, Wang Q, Wei S, et al: A MALAT1/HIF-2 $\alpha$ feedback loop contributes to arsenite carcinogenesis. Oncotarget 7: 5769-5787, 2016.

23. Panzitt K, Tschernatsch MM, Guelly C, Moustafa T, Stradner M, Strohmaier HM, Buck CR, Denk H, Schroeder R, Trauner M and Zatloukal K: Characterization of HULC, a novel gene with striking up-regulation in hepatocellular carcinoma, as noncoding RNA. Gastroenterology 132: 330-342, 2007.

24. Ohrnberger S, Thavamani A, Braeuning A, Lipka DB, Kirilov M, Geffers R, Autenrieth SE, Römer M, Zell A, Bonin M, et al: Dysregulated serum response factor triggers formation of hepatocellular carcinoma. Hepatology 61: 979-989, 2015.

25. Li J, Chen Z, Tian L, Zhou C, He MY, Gao Y, Wang S, Zhou F, Shi S, Feng X, et al: LncRNA profile study reveals a three-lncRNA signature associated with the survival of patients with oesophageal squamous cell carcinoma. Gut 63: 1700-1710, 2014.

26. Thum T: Noncoding RNAs and myocardial fibrosis. Nat Rev Cardiol 11: 655-663, 2014.

27. Zhang S, Chen S, Yang G, Gu F, Li M, Zhong B, Hu J, Hoffman A and Chen M: Long noncoding RNA HOTAIR as an independent prognostic marker in cancer: A meta-analysis. PLoS One 9: e105538, 2014.

28. Carr BI, Guerra V, Giannini EG, Farinati F, Ciccarese F, Rapaccini GL, Di Marco M, Benvegnù L, Zoli M, Borzio F, et al: Significance of platelet and AFP levels and liver function parameters for HCC size and survival. Int J Biol Markers 29: e215-e223, 2014.

29. Dwyer JP, Hosking P and Lubel J: Multiple liver lesions in a patient with positive hepatitis C serology and elevated AFP: Is it HCC? Gastroenterology 147: e12-e13, 2014

30. Li T, Xie J, Shen C, Cheng D, Shi Y, Wu Z, Deng X, Chen H, Shen B, Peng C, et al: Upregulation of long noncoding RNA ZEB1-AS1 promotes tumor metastasis and predicts poor prognosis in hepatocellular carcinoma. Oncogene 35: 1575-1584, 2016.

31. Li T, Xie J, Shen C, Cheng D, Shi Y, Wu Z, Deng X, Chen H, Shen B, Peng C, et al: Amplification of long noncoding RNA ZFAS1 promotes metastasis in hepatocellular carcinoma. Cancer Res 75: 3181-3191, 2015. 